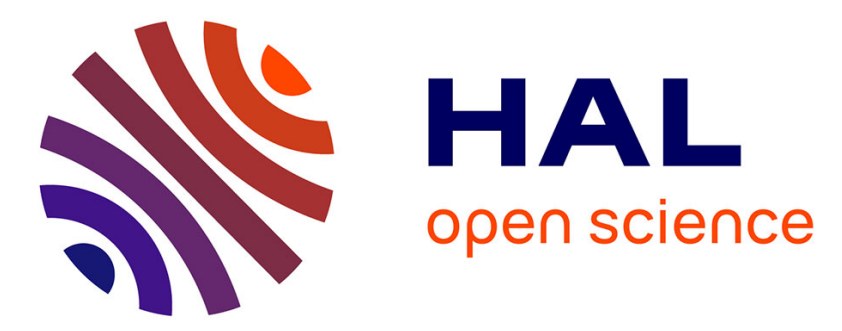

\title{
Les politiques chinoises de villes nouvelles : trajectoire et ajustements de l'action publique urbaine à Shanghai
}

Carine Henriot

\section{To cite this version:}

Carine Henriot. Les politiques chinoises de villes nouvelles : trajectoire et ajustements de l'action publique urbaine à Shanghai. Géocarrefour - Revue de géographie de Lyon, 2015, 90 (90/1), pp. 27 38. 10.4000/geocarrefour.9659 . hal-01865057

\section{HAL Id: hal-01865057 https://hal.science/hal-01865057}

Submitted on 30 Aug 2018

HAL is a multi-disciplinary open access archive for the deposit and dissemination of scientific research documents, whether they are published or not. The documents may come from teaching and research institutions in France or abroad, or from public or private research centers.
L'archive ouverte pluridisciplinaire HAL, est destinée au dépôt et à la diffusion de documents scientifiques de niveau recherche, publiés ou non, émanant des établissements d'enseignement et de recherche français ou étrangers, des laboratoires publics ou privés. 


\section{Carine HENRIOT}

Sorbonne Universités - Université de Technologie de Compiègne

\title{
Les politiques chinoises de villes nouvelles : trajectoire et ajustements de l'action publique urbaine à Shanghai
}

\author{
RÉSUMÉ \\ Cette contribution analyse l'action publique urbaine chinoise à partir des politiques de villes nou- \\ velles, en combinant l'étude, sur le temps long, des modèles de villes nouvelles aménagées à \\ Shanghai, et leurs ajustements récents. L'article démontre qu'il existe une succession du modèle de \\ ville nouvelle (ville-satellite, ville nouvelle, ville durable), alimentée par la circulation de références \\ urbaines internationales. La diversification des modèles de ville durable (ville verte, éco-ville, ville \\ bas-carbone) est représentative de l'inflexion de l'action publique urbaine chinoise vers la transition \\ écologique et énergétique. Enfin, à une action publique urbaine descendante se substituent des \\ initiatives plurielles, portées par l'État, les collectivités territoriales - municipale et d'arrondisse- \\ ment -, les PPP, et leur mise en concurrence.
}

\begin{abstract}
The article analyzes urban governance in China through the study of new town policy by combining the long term study of their development at Shanghai with more recent adjustments to them. The paper demonstrates that there has been a succession of new town models (satellite towns, new cities, sustainable cities) feeding off international experience of urban development. The current diversification of sustainable city models (green cities, eco-cities, low-carbon cities) is representative of a shift in Chinese urban governance towards environmental and energy transitions. Finally, top-down urban governance is being replaced by diverse public and private initiatives, supported by the State, local authorities - towns and districts -, and PPPs, and by competition between them.
\end{abstract}

En Chine, si l'action publique urbaine et sa révision sur le temps long ne font pas directement I'objet de publications, I'urbanisation chinoise, ses leviers économiques, ses rythmes et ses formes de territorialisation sont quant à eux connus (Kirkby, 1987 ; Ma, 2002 ; Pannell, 2002 ; Friedman, 2006 ; Chan, 2010). La transition urbaine chinoise s'éloigne du modèle de transition urbaine de Lewis (1954) pour strictement limiter la croissance urbaine et n'encourager que le développement de secteurs industriels. Puis I'urbanisation chinoise s'éloigne des modèles de transition observés dans les pays de l'ancien bloc soviétique et d'Europe de l'Est, en raison de la rupture de la Chine avec I'URSS en 1960, pour défendre un modèle de développement socialiste chinois alternatif au modèle soviétique ( $\mathrm{Ma}, 2002)$, où l'industrialisation s'accompagne d'une extension urbaine limitée, les programmes de logements ouvriers. Ensuite, la transition urbaine chinoise ne s'inscrit pas en rupture avec le communisme, même après la réintroduction du marché, et ne fait pas suite à une crise. Bien au contraire, elle s'inscrit dans une continuité politique et correspond à des " réformes par le haut " (Bergère, 1985, p. 157), initiées par l'État central. L'ouverture aux investissements étrangers s'effectue graduellement : d'abord au sein de zones économiques spéciales (ZES) dans le sud de la Chine en 1980 ; puis sous la forme de zones de développement économique et technologique (ZDET) en 1984 ; avant que les principales villes littorales soient ouvertes en 1985 - autant de projets pilotes faisant partie intégrante de la voie chinoise du changement économique. Une décentralisation des pouvoirs de décision économique au profit des provinces, des municipalités et de leurs collectivités territoriales, orga- nise l'industrialisation rurale prospère des régions littorales. Dans les années 1990, de nouvelles logiques d'acteurs apparaissent. La ville chinoise devient, l'organisateur, à son profit, du développement économique, l'aménageur des bourgs industriels et des périphéries rurales (Sanjuan, 2000 , p. 145). La gouvernance métropolitaine est désormais assurée par les gouvernements municipaux, tandis que les milieux d'affaires investissent la production urbaine (Lin et Wei, 2002, p. 15371538). L'arrivée des opérateurs internationaux entraîne des ajustements structurels, favorise une gestion urbaine décentralisée et de nouvelles formes de planification dominées par la logique de projet, notamment dans les grands équipements, les services et les réseaux urbains (Lorrain, 2010; Lorrain 2011 ; Zhang, 2014 ; Zhang et al., 2015). La ville chinoise construite dans les années 2000 se caractérise par de vastes opérations de renouvellement urbain, la verticalisation de son paysage urbain et un redéploiement métropolitain polycentrique. Aussi, transition urbaine, action publique urbaine et formes urbaines chinoises sont-elles étroitement corrélées et conditionnées par les enjeux territoriaux et les orientations sectorielles fixés par les décideurs au pouvoir.

Dès lors, le projet urbain traduit les choix de l'action publique en termes de référentiels, d'instruments de la production urbaine et de formes de territorialisation, ce qui interroge le rôle de l'expertise nationale et internationale, de la circulation des modèles de développement urbain, comme celui des villes nouvelles. En effet, par opposition à un développement urbain " au fil de l'eau ", la création d'une ville nouvelle constitue un " processus d'urbanisme raisonné ", décidé par voie 
administrative, généralement dans le cadre d'une politique d'aménagement et qui marque le degré le plus élevé de I'intervention publique (Merlin, 1972 , p. 355 ; Chaline, 1996, p. 20-28 ; Merlin, 1996, p. 847-850). Aussi, la ville nouvelle, en tant qu'outil de planification territoriale, cristallise I'action publique urbaine.

Cet article analyse en particulier les politiques shanghaiennes de villes nouvelles pour nourrir cette réflexion sur l'action publique dans une métropole émergente chinoise. Le cas des villes nouvelles de Shanghai est emblématique du changement sociétal observé dans les pays émergents (Houssay-Holzschuch, 2010, p. 12). II illustre la mise en spectacle des recompositions accélérées des actions publiques urbaines chinoises, et particulièrement de la métropole shanghaienne et par ses villes nouvelles. Cette contribution combine également deux prises de recul. Sur le temps long, elle s'intéresse à la succession des modèles de villes nouvelles mobilisés, en résonnance avec les mutations urbaines accélérées observées à Shanghai au début du XXle siècle. Les politiques chinoises de villes nouvelles, leurs implantations, leurs référentiels et formes de territorialisation seront ainsi analysés dans un premier temps, avant un focus sur les politiques de villes nouvelles dans la municipalité de Shanghai. Enfin, ces transitions seront confrontées aux ajustements récents des politiques de villes nouvelles à Shanghai. Selon ces inflexions de plus court terme, on s'attachera à la diversification des acteurs qui produisent I'urbain, à travers le projet d'éco-ville de Dongtan.

\section{LA TRAJECTOIRE DES POLITIQUES DE VILLES NOUVELLES EN CHINE}

Suivant les phases de la transition urbaine chinoise, I'action publique urbaine privilégie différentes formes de territorialisation, aménagées dans le cadre de politiques de villes nouvelles. Nous faisons I'hypothèse que la ville-satellite, la ville nouvelle et la ville durable renvoient à une même volonté politique d'orienter la croissance urbaine vers un développement maîtrisé. Cette terminologie différenciée correspond, selon nous, aux différentes phases de la transition urbaine chinoise (Henriot, 2013b, p. 63-90), soit à des objectifs et à des modes de territorialisation différenciés, bénéficiant inégalement de l'ouverture/ fermeture aux modèles internationaux d'urbanisme et d'aménagement de l'espace. Alimentés par la circulation de modèles de développement urbain mondialisés et leur appropriation par les acteurs nationaux et locaux, trois modèles de villes nouvelles se succèdent en Chine, entre 1949 et nos jours.

\section{Les villes-satellites chinoises}

Le modèle soviétique de ville-satellite est intro- duit en Chine à l'occasion du ler plan quinquennal $(1955-1957)^{1}$ (Hoa, 1981, p. 63-65 ; Petit, 2002, p. 81-86). Suivant la logique des fronts pionniers soviétiques en Sibérie ou en Extrême-Orient russe, les villes nouvelles industrielles chinoises sont construites sur les marches de la Chine, s'inscrivent dans une politique de conquête d'un vaste territoire, et visent l'exploitation de ressources naturelles (Kirkby, 1987, p. 205).

Dans le cadre du $\|^{\mathrm{e}}$ plan quinquennal (19581962), les villes littorales chinoises sont l'objet d'un redéploiement industriel vers leurs périphéries. Ce programme de villes-satellites se présente comme une alternative raisonnée au problème de croissance des très grandes villes. Sur le modèle du développement soviétique, il prévoit la délocalisation en périphérie des projets industriels de grande échelle (Kirkby, 1987, p. 207). De nombreuses villes-satellites industrielles, juxtaposées les unes aux autres, se développent alors autour de Hefei, Zhengzhou, Tianjin, Nankin, Jinan, Handan, Changchun, Pékin, Canton, Changsha, Daqing et Shanghai (Funk, 1996, p. 338). Cette première période de création de villes nouvelles correspond à la phase d'industrialisation de la Chine sans extension urbaine, et se traduit par le développement de villes-satellites destinées à rationaliser et à optimiser le développement industriel soutenu. Ces villes-satellites sont construites en continuité avec les bourgs-centres administratifs gravitant autour de I'agglomération et s'appuyant sur des axes de transport, comme les voies ferrées, les voies d'eau et les routes principales. Autour de ces extensions mono-industrielles se juxtaposent, comme pour les villes nouvelles de I'Union soviétique et des pays socialistes européens, des zones résidentielles destinées aux travailleurs.

Après 1978, I'industrialisation rurale favorise le développement de ces villes-satellites, qui connaissent un nouvel essor dans les années 1980. Cependant, les aménageurs manquent d'expérience pour améliorer ce programme de redéploiement territorial. Avec le départ des experts soviétiques en 1960 et en raison de l'isolement que connaît la Chine jusqu'à la fin des années 1980, la planification des villes-satellites ne peut s'appuyer sur l'expérience internationale (Hoa, 1981, p.63-65).

\section{Les villes nouvelles contemporaines}

Au début des années 1990, les projets de développement des grandes agglomérations chinoises adaptent le modèle des villes-satellites dans un contexte d'ouverture à l'expertise internationale, de réouverture du marché de l'immobilier notamment résidentiel, et de modernisation urbaine.

La profession d'architecte-urbaniste-aménageur, longtemps discréditée, est réhabilitée.
1 - Le premier plan quinquennal est adopté en 1955 et considéré comme rétroactif (1953-1957). Toutefois, ce plan quinquennal est véritablement mis en place durant 3 ans (1955-1957). 
Si les Universités Tsinghua à Pékin et Tongji à Shanghai sont les seules universités chinoises à dispenser l'enseignement de ces disciplines durant la Révolution culturelle (Hoa, 1981, p. 6365), I'ouverture des années 1980 autorise puis encourage les premiers échanges scientifiques et professionnels : I'expertise urbaine internationale est réintroduite en Chine. Des institutions étrangères cherchent à mettre en œuvre une politique d'aide au développement, à mettre en avant leur expertise nationale pour favoriser l'ouverture de nouveaux marchés. Dans le champ de l'urbanisme et de l'aménagement, des visites officielles de délégations étrangères publiques et parapubliques se multiplient, comme en témoigne l'exemple des relations sino-françaises. Dès 1984, la mission interministérielle de l'information scientifique et technique (MINIST) française, accompagnée de I'Institut d'aménagement et d'urbanisme de la région Île-de-France (IAURIF), en charge de la veille et de la prospective pour les villes nouvelles franciliennes, rencontrent les instituts d'information scientifique et technique des municipalités de Pékin et de Shanghai (Henry, 1984, p. 65-70). À la fin des années 1980, des échanges réguliers, bien que difficiles, sont entretenus entre I'IAURIF et les responsables en charge de la planification des villes-satellites de Shanghai (Antier et Abadia, 1987 ; Antier, 1989) et de Pékin (Etteinger, 1992). Au début des années 1990, les premiers cabinets $d$ 'architecture et d'urbanisme étrangers s'implantent en Chine, comme les cabinets français Arté-Charpentier ou ADP Paul Andreu. Ces acteurs français, publics et privés, en mobilisant l'expérience des villes nouvelles françaises, participent à la transmission du modèle français de villes nouvelles et permettent aux acteurs chinois de se réapproprier les schémas mais aussi les outils de l'urbanisme réglementaire, la planification et l'urbanisme opérationnel (Petit, 2002, p. 21-43).

Les villes nouvelles chinoises de cette seconde génération répondent à de nouvelles exigences. Elles ne se caractérisent plus par une mono-fonctionnalité industrielle affirmée, mais au contraire par leur multifonctionnalité : activités industrielles et activités tertiaires, fonction résidentielle et fonction récréative sont désormais représentées (Billard, 2005). Ces nouvelles entités urbaines sont pensées de manière systémique et doivent participer au développement d'ensemble de la région métropolitaine. Elles visent une certaine autonomie à l'échelle de la ville nouvelle (écoles, hôpitaux), tout en s'inscrivant en complémentarité fonctionnelle et morphologique à l'échelle de l'aire métropolitaine (antennes universitaires déconcentrées, zones d'activités, parcs de récréation, offre résidentielle diversifiée). Initialement conçues comme des espaces de desserrement des agglomérations et de rationalisation de leurs développements périphériques ; elles participent à la fois à la redis- tribution des activités et à celle de la population (Henriot, 2013b, p. 171-201). Elles répondent enfin à de nouvelles exigences en termes de rationalisation de l'espace urbain et de valorisation par l'image. Les villes nouvelles chinoises se verticalisent pour devenir les vitrines de la modernité et le symbole des pouvoirs en place (Baudon, 2002).

\section{L'émergence du concept de ville durable en Chine}

Au début des années 2000, la Chine connaît une intégration accélérée à la globalisation et s'insère sur la scène internationale. Or la préservation de I'environnement, le développement urbain durable et la lutte contre le changement climatique mobilisent les grandes puissances internationales, et participent de leur influence. En 2002, le gouvernement central chinois créée un ministère de l'Environnement. En 2006, dans le cadre du Xle plan quinquennal (2006-2010), les politiques de développement urbain sont infléchies pour désormais prendre en considération l'environnement. Le gouvernement central nourrit alors I'ambition d'élaborer un modèle chinois de ville durable. II mobilise pour cela l'ensemble des acteurs à sa disposition et les positionne en situation de concurrence. Ainsi les initiatives se multiplient-elles : le paradigme de l'éco-construction est exploré par le ministère du Logement et du développement urbain et rural à travers le label "éco-cité-jardin " (shengtai yuanlin chengshi), initié en 2004, dont les projets pilotes sont identifiés en 2006. Le référentiel de la ville verte est privilégié quant à lui par le ministère de la Protection environnementale, qui valorise le label " éco-ville " (shengtai chengshi) en 2007, tandis que celui de la "ville bas-carbone " (ditan chengshi) est promu par la Commission nationale pour le développement et les réformes à partir de 2010 (Baeumler et al., 2012 ; Zhou et al., 2012 ; Henriot, 2013b, p. 81-86).

La Chine encourage également le développement d'un quatrième type de villes durables, portées par les coopérations internationales et bilatérales. Les projets conçus dans le cadre de partenariats internationaux sont ambitieux et largement médiatisés comme "Caofeidian International Eco-City " à Tangshan. Toutefois, il convient de se montrer prudent quant à la durabilité de ces projets. Si la ville écologique sino-singapourienne de Tianjin, "Sino-Singapour Tianjin Eco-City » (STEC), lancée en novembre 2007 à des fins économiques, fait l'objet d'évaluations positives par les bailleurs de fonds comme la Banque mondiale (2009) ou la Banque asiatique de développement (2010), I'initiative sino-singapourienne développée à Suzhou, le "Suzhou Industrial Park " (SIP) est qualifié de planification urbaine " hyper-productiviste et fonctionnaliste " éloignée des préoccupations environnementales (Curien, 2014). Dans le cadre de l'expérimentation du modèle bas-carbone dans la province pilote du Hubei, la France développe, 


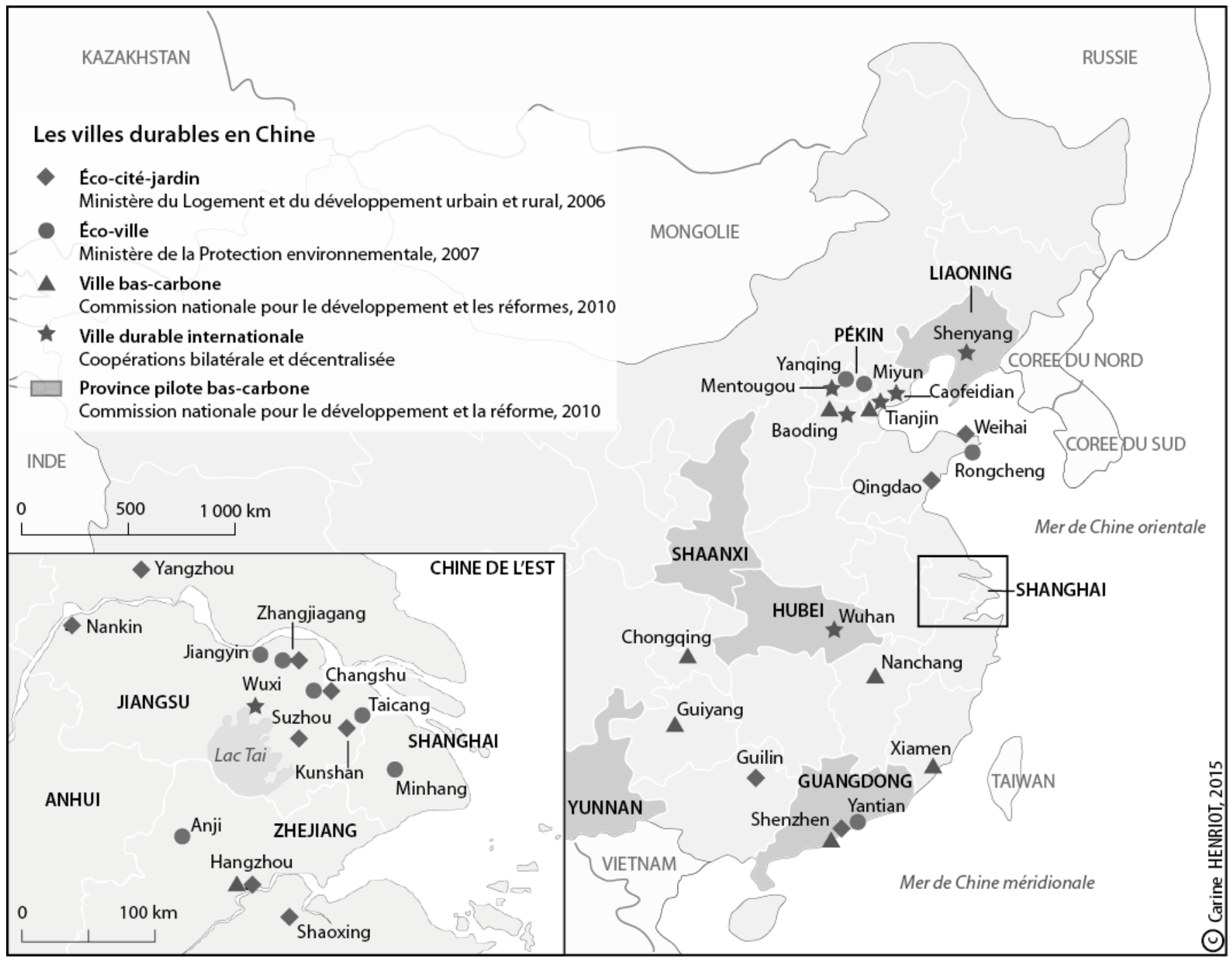

conjointement avec les autorités provinciales et municipales chinoises, un projet de ville durable à Wuhan.

Ainsi, la succession de ces modèles de villes nouvelles témoigne du rôle de l'expertise internationale et de la circulation des modèles au sein de la trajectoire suivie par l'action publique urbaine chinoise, selon plusieurs phases et des processus de territorialisation différenciés. Une étude fine des politiques de villes nouvelles à Shanghai permet de mieux comprendre ces enjeux territoriaux et les logiques d'acteurs sous-jacentes.

\section{LA TRAJECTOIRE DES POLITIQUES DE VILLES NOUVELLES À SHANGHAI}

Shanghai est une municipalité élevée au rang de province dans la Chine de l'Est, c'est-à-dire directement placée sous l'autorité du pouvoir central. À la fin de 2014, la municipalité accueille plus de 24,2 millions d'habitants, dont 9,9 millions de résidants temporaires, sur un territoire de $6340 \mathrm{~km}^{2}$ (BSS, 2015). Si les politiques shanghaiennes de villes nouvelles suivent les conditions d'aménagement des grandes agglomérations littorales, leur rythme de développement y est plus saccadé. Les formes de la métropolisation, adaptées de modèles d'aménagement urbain internationaux, sont également mieux dessinées.
Les villes-satellites shanghaiennes

Lors de l'instauration de plans de développement quinquennaux, sur le modèle soviétique, Shanghai reste à l'écart des politiques d'industrialisation fixées par le gouvernement central de Pékin. Adopté en 1955, le premier plan quinquennal de Shanghai (1953-1957) vise à transférer l'industrie lourde de la ville de Shanghai vers la Chine intérieure, et à l'échelle municipale en direction des périphéries rurales, tout en limitant leur urbanisation.

L'aménagement des périphéries de Shanghai débute avec la redéfinition administrative du cadre d'intervention municipale. En 1958, dix districts adjacents, initialement inclus dans la province du Jiangsu, sont agrégés. La Municipalité gère alors un territoire de $5910 \mathrm{~km}^{2}$, justifié par la complémentarité de fonctions urbaines et rurales, et administre quelques dix millions d'habitants. $C^{\prime}$ est à cette échelle que sont planifiés plusieurs programmes d'aménagement des transports et de ravitaillement agricole, tandis que des villessatellites sont planifiées pour accompagner la déconcentration de la population urbaine, celle des industries, et améliorer la productivité (Fung, 1981a et b ; Bergère, 2002 ; Kirkby, 1987). Le programme de villes-satellites mono-industrielles est adopté puis rendu officiel en 1959. Cinq sites sont retenus en proche banlieue : Minhang,
Figure 1 : les villes durables chinoises et ses modèles urbains

Sources : - Ministère du Logement et du développement urbain et rural, http://www.mohurd.gov.cn/

- Ministère de la Protection environnementale, http:// english.mep.gov.cn/

- Commission pour les réformes et le développement, http://en.ndrc.gov.cn/

- Banque mondiale

- Banque asiatique de développement, 2010. 
Wujing, Jiading, Songjiang et Anting. Dans les années 1970, Jinshanwei et Baoshan viennent compléter le dispositif satellitaire. Aucun programme d'ensemble ne vient coordonner le développement des anciennes villes-satellites de 1958 et des deux nouvelles villes-satellites développées dans les années 1970-1980 (Ning et Yan, 1995, p. 585).

Autour de ces extensions mono-industrielles se juxtaposent, comme pour les villes nouvelles de I'Union soviétique et des pays socialistes européens, des zones résidentielles destinées aux travailleurs. Cette seconde forme d'extension urbaine apparaît sous le toponyme xincun, " nouveau village ", et voit les banlieues résidentielles s'étendre avec monotonie sous la forme de vastes quartiers constitués de blocs d'habitations répétitifs de trois ou cinq étages (Ged, 2000, p. 35 ; Bergère, 2002, p. 398). Ces ensembles sont construits par le bureau de district ou d'arrondissement du travail, puis par les unités de travail (danwei), pour loger les ouvriers employés et leur famille, la main-d'œuvre nécessaire à l'effort $d^{\prime}$ 'industrialisation du pays. Peu à peu, la société chinoise, et par extension les villes chinoises, se transforment pour s'organiser autour et à partir des lieux de production. Un faible intérêt est alors porté au logement, aux équipements et aux services urbains. Cette organisation demeure jusqu'à la fin des années 1980 .

Ainsi, durant les trente premières années du régime socialiste, mis à part l'expansion urbaine en " tâche d'huile ", l'action publique urbaine chinoise est-elle dominée par la création de villessatellites, associant deux types d'extension périurbaine, les complexes industriels et les " nouveaux villages " résidentiels aux équipements et services modestes, en raison de la faiblesse des investissements non productifs.

\section{Les villes nouvelles shanghaiennes contemporaines}

La modernisation urbaine des années 1990 transforme profondément le paysage urbain de la villecentre de Shanghai, en construisant au-delà de la rivière Huangpu le nouveau quartier de Pudong qui accueille, sur $520 \mathrm{~km}^{2}$ le centre d'affaires de Lujiazui, la zone de libre-échange de Waigaochao, celle d'import-export de Jinqiao, le parc industriel de nouvelles technologies de Zhangjiang, le parc d'agriculture moderne de Sunqiao, la cité administrative de la Municipalité, ainsi que de vastes secteurs résidentiels. Pour autant, Pudong n'est pas considéré comme une ville nouvelle, mais comme le " nouveau secteur cristallisant la modernisation " à Shanghai (xiandaihua xinqu) (Yu, 2010, p. 19-21).

Dans le cadre du schéma directeur de 19992020, plusieurs programmes de villes nouvelles sont développés à Shanghai. Ils favorisent un redéploiement polycentrique et hiérarchisé de l'espace métropolitain et participent à l'intégration des territoires nouvellement urbanisés à la métropole shanghaienne. Cinq programmes de villes nouvelles se succèdent entre 1999 et 2013 : ils sont ajustés en fonction des politiques nationales, des orientations établies par les plans quinquennaux, des priorités et des objectifs fixés par la Municipalité et les autorités d'arrondissements, et de leur mise en œuvre par les gouvernements $d$ 'arrondissement. Le schéma directeur de Shanghai publié en 1999 et approuvé en 2001 contient un plan d'aménagement de l'armature urbaine à l'échelle de la municipalité, ainsi qu'un plan de relance des villes-satellites qui sont désormais appelées "villes nouvelles " (xincheng). Onze villes nouvelles de 200000 à 300000 habitants sont ainsi planifiées et inégalement aménagées durant les trois plans quinquennaux suivants. Dans la cadre du $X^{e}$ plan quinquennal (2001-2005), dont le slogan est « urbaniser la banlieue ", un projet expérimental " une ville, neuf bourgs " (yi cheng jiu zhen) est lancé en 2001. II vise la construction de dix "bourgs expérimentaux " (shidian zhen), des villes nouvelles de petite dimension, présentant une composition urbaine et un style architectural à thème : allemand, anglais, italien, etc. En 2003, le plan de construction urbaine à court terme à Shanghai (2003-2007), qui phase et ajuste le schéma directeur de Shanghai (1999-2020), maintient le projet pilote « une ville, neuf bourgs " et préconise de développer en priorité " trois villes nouvelles majeures " (san da xincheng) : Songjiang, Jiading-Anting et Lingang. Ces trois villes nouvelles sont alors planifiées pour accueillir chacune 800000 à 1 million d'habitants. En janvier 2006, dans le cadre du Xle programme d'action quinquennale (2006-2010), est lancé le projet de redéploiement polycentrique hiérarchisé « 1-9-6-6 " qui réaffirme l'aménagement de neuf villes nouvelles, les trois villes nouvelles majeures, ainsi que six villes nouvelles secondaires de 300000 habitants (Yu et Luo, 2009, p. 15). Enfin, dans le cadre du XIle programme d'action quinquennal, sept villes nouvelles sont aménagées et distinguées à travers l'appellation ville nouvelle de premier, de second et de troisième rang. La ville nouvelle de Songjiang est planifiée pour accueillir à I'horizon 2020 entre 200000 et 400000 habitants, sur $120 \mathrm{~km}^{2}$. Ainsi entre 1999 et 2015, cinq programmes de redéploiement polycentrique se succèdent : ils ajustent, hiérarchisent et phasent le développement des villes nouvelles de Shanghai, mais confirment, à chaque nouveau programme, la polarité de Songjiang, Jiading et Lingang-Nanhui.

Ces villes nouvelles sont désormais de véritables pôles multifonctionnels de desserrement de la municipalité. Ils adossent à des polarités rurales anciennes, voire des villes-satellites, de vastes 


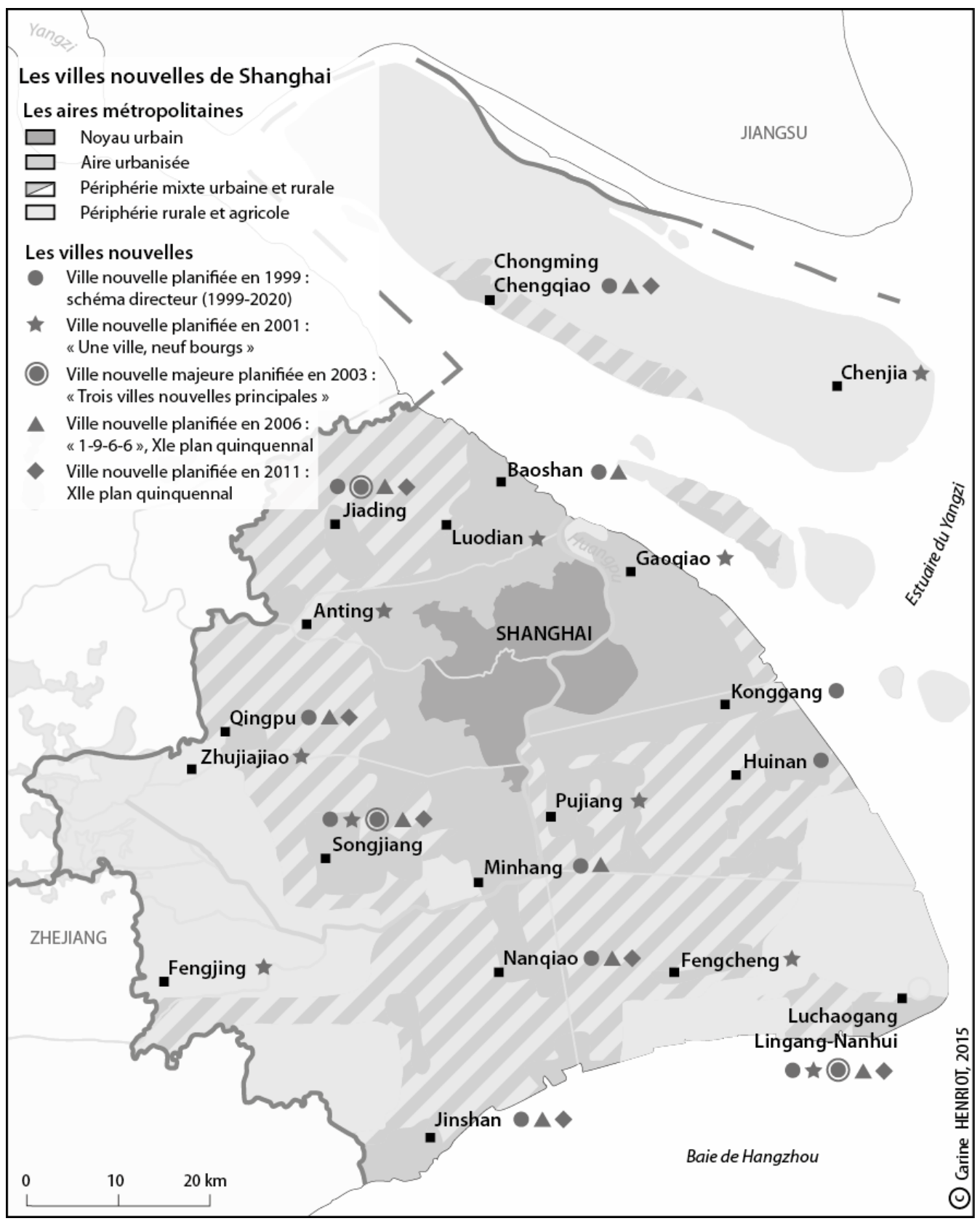

secteurs multifonctionnels aménagés suivant les principes du fonctionnalisme introduits dans les années 1990 (Zhuo, 2004), qualifié également $d^{\prime}$ " hyper-fonctionnalisme " (Curien, 2014), ou de " modèle intégrationniste " (Doulet, 2015), couplés au principe du transit-oriented development (TOD) (Zhuo, 2015). Ce zonage aménage une ville souhaitée plus efficace dans son fonctionnement. Toutefois, contrairement au modèle collectiviste des unités de travail, qui associait sur un même lot I'usine, les logements du personnel et l'ensemble des commerces et services destinés aux habitants, la différenciation des espaces et leur spécialisation fonctionnelle induisent des déplacements et des mobilités croissantes : les distancetemps ne cessent de croître. La rationalisation de la trame et du tissu urbains inscrit le zonage fonctionnel et les nouvelles mobilités générées dans un plan orthogonal orienté nord-sud et est-ouest. De larges artères se coupant à angle droit et dont le gabarit répond aux nouvelles règles d'urbanisme, séparent strictement les usagers (quatre roues/ deux roues/piétons) donnant incontestablement I'avantage à l'automobile. L'ensemble articule de vastes secteurs résidentiels de grands collectifs
Figure 2 : Les programmes de villes nouvelles à Shanghai entre 1999 et 2015

Source pour le fond de carte et les aires métropolitaines :

- Monin É., 2012 :

Source pour les figurés, localisant les programmes de villes nouvelles: Yu S. et Luo Z. 2009 ; Shanghai tongji chengshi guihua sheji yanjiu yuan [Institut d'urbanisme de Tongji à Shanghai], 2011. "Shi'erwu shanghai jiaoqu xincheng guihua » [Planification des villes nouvelles du XII ${ }^{\mathrm{e}}$ programme d'action quinquennal de Shanghai], p.4. 
2 - Parc de la zone humide de Dongtan, http://www. dongtanwetland.com.

3 - Visites de terrain le $15 / 03 / 2008$ et le $12 / 03 / 2011$. aménagés par des sociétés de développement associant la Municipalité au secteur privé, en dehors des schémas directeurs des villes nouvelles, pour les segments non-solvables de la population, ce qui introduit une macro-ségrégation résidentielle (Henriot, 2014).

Au fil du temps, le concept de ville nouvelle a été infléchi et les termes employés pour désigner de grandes opérations de construction résidentielle de redéploiement polycentrique ont varié (villes-satellites, villes nouvelles ou nouveaux bourgs, villes nouvelles majeures ou secondaires, " grandes communautés résidentielles »). À travers ces laboratoires de l'urbain, les autorités locales cherchent, à l'échelle intra-urbaine, à répondre aux défis posés par la métropolisation comme la congestion urbaine, le redéploiement, I'intégration territoriale, la cohésion sociale, tout en positionnant la ville sur la scène internationale. Dans un contexte d'ouverture, de globalisation et de mondialisation, les objectifs poursuivis par ces programmes de villes nouvelles nous renseignent également sur l'appropriation des modèles urbains internationaux, sur la diffusion de standards internationaux et l'injonction, plus récente, au développement urbain durable.

\section{LES AJUSTEMENTS RÉCENTS DE L'ACTION PUBLIQUE URBAINE À DONGTAN}

Bien que les ministères de l'Environnement et du Logement aient lancés plusieurs programmes de villes durables, les villes nouvelles aménagées à Shanghai dans les années 2000 ne sont pas les réceptacles privilégiés de projets d'écoconstruction. Si la conception de plusieurs quartiers présente une morphologie urbaine optimisée, comme Xin Jiangwan à Yangpu (Wang, 2011), le projet d'éco-ville de Dongtan a connu de nombreux rebondissements, révélateurs des ajustements récents de l'action publique urbaine à Shanghai.

\section{L'île écologique et le secteur de Dongtan}

Située au nord de la municipalité de Shanghai dans l'embouchure du Yangzi, Chongming est une île alluviale de $1000 \mathrm{~km}^{2}$ qui s'accroît de quelques mètres carrés par an. À l'extrémité sud-orientale de l'île, la " première ville entièrement écologique " de Chine, l'éco-ville de Dongtan (dongtan shengtai cheng) est " en cours de construction" depuis une quinzaine $d$ 'années.

En 2001, le nouveau bourg de Chenjia, développé dans le cadre du projet " une ville, neuf bourgs", constitue une première réflexion du projet de ville écologique de Dongtan. II a été initialement conçu selon le "style écologique de l'île verte " sur une surface de $22 \mathrm{~km}^{2}$, en harmonie avec la réserve ornithologique et la zone humide de Dongtan (Wang et Li, 2006, p. 8-11). En 2002, Chongming est le dernier district de la municipalité où l'espace rural domine encore. II est retenu par le futur ministère de l'Environnement chinois pour devenir une zone écologique pilote (Huang et al., 2008, p. 576). En 2003, le parc de la zone humide de Dongtan ${ }^{2}$ est aménagé par la Société d'investissements industriels de Shanghai (SIIC - Shanghai Industrial Investment Holdings Corporation), dont la filiale immobilière locale, la Société de développement et d'investissement de Dongtan (Shanghai shiye dongtan touzi kaifa youxian gongsi), détient depuis 1998 les droits d'usage du sol (Shen et Wu, 2012, p. 236). En 2004, suite à des propositions de I'Institut McKinsey et après une compétition internationale, la SIIC confie au consultant britannique ARUP la stratégie de développement et d'aménagement "zéro carbone " du secteur de Dongtan, sur $86 \mathrm{~km}^{2}$. Le schéma directeur prévoit notamment une zone naturelle humide mise en valeur pour le tourisme, une zone d'agriculture biologique et une éco-ville. En 2005, la conception détaillée de nombreux bâtiments est confiée à des entreprises chinoises, qui sont chargées de " siniser " le projet d'île écologique et d'éco-ville (Den Hartog, 2010, p. 160-171). La première phase de l'éco-ville aménagée sur $6,3 \mathrm{~km}^{2}$ est composée de trois secteurs appelés "villages" dédiés au tourisme de récréation, à l'innovation technologique et à la santé. En novembre 2005, conscients de l'innovation conceptuelle que représente Dongtan, la SIIC, le président Hu Jintao et le premier ministre britannique Tony Blair signent, en marge d'un accord commercial, une déclaration de coopération pour le développement de l'éco-ville de Dongtan (Den Hartog, 2010 ; Wu, 2012). En 2006, le projet est gelé et officiellement abandonné en 2008. Les pages dédiées au projet sur le site internet d'ARUP, ainsi que le site officiel d'éco-ville de Dongtan, sont supprimés de la toile. En janvier 2010, un nouveau programme pour l'île de Chongming : "Chongming Eco-Island Construction Outline 2010-2020 " est publié et approuvé en mars par le ministère des Sciences et technologies. En avril 2010, juste avant le lancement de I'exposition universelle de Shanghai, la zone humide de Dongtan est réaménagée pour accueillir un vaste parc dédié à l'éco-tourisme. Proche des éco-musées français, le parc éveille à I'environnement, à sa connaissance et à son respect, tout en proposant des activités ludiques; le visiteur peut se promener à pied, en bicyclette, en voiture électrique ou en bateau électrique au sein de cette zone moins de préservation que de récréation ${ }^{3}$. Au niveau municipal, le projet d'île écologique de Dongtan est confirmé dans le cadre du « Douzième programme d'action et de développement quinquennal : rendre l'environnement de la ville plus propre et plus plaisant " (GMS, 2011). En 2012, la presse chinoise se fait l'écho d'un nouveau plan d'aménagement du secteur de Dongtan, ainsi que du plan détaillé de l'éco-ville (Zang, 2012). En 2015, seul le parc de récréa- 
tion de la zone humide de Dongtan est aménagé ; l'éco-ville reste à l'état de projet.

\section{Les ajustements du projet d'éco-ville}

Plusieurs observateurs non-gouvernementaux dénoncent, depuis 15 ans, I'aménagement d'une éco-ville à proximité d'une zone protégée Ramsar servant d'habitat au printemps et à l'automne pour 2 à 3 millions d'oiseaux migrateurs sur leur route entre I'Asie et I'Australie. Toutefois, ce $n^{\prime}$ est pas pour des raisons écologiques, mais pour des raisons politiques et économiques que le projet de Dongtan connaît de nombreux rebondissements depuis 2006, est officiellement abandonné en 2008, puis relancé en 2012, sans toutefois qu'aucun aménagement ne soit réalisé à ce jour. Ces allers et retours sont révélateurs des ajustements récents de I'action publique urbaine shanghaienne.

Le projet d'éco-ville de Dongtan était largement soutenu par Chen Liangyu, I'ancien secrétaire du Parti communiste de la municipalité de Shanghai, soit le " numéro 1 " de la Municipalité entre octobre 2002 et septembre 2006, avant qu'il ne soit démis de ses fonctions puis condamné, en avril 2008, à 18 ans de prison, pour détournement de fonds publics, abus de pouvoir et corruption. Si la presse internationale explique I'abandon du projet de Dongtan par la déchéance de l'ancien dirigeant de Shanghai, la réalité est plus complexe.

L'un des enjeux auxquels le projet de Dongtan est confronté dès le début est le quota de surfaces constructibles et de terres agricoles. Afin de conserver son autonomie alimentaire, la Chine a gelé 1,8 milliard de mu (soit 120 millions d'hectares) de terres arables destinées à l'exploitation agricole. Le ministère chinois des Ressources et du sol est chargé d'allouer à chaque gouvernement local un quota de surfaces constructibles et de terres agricoles. Chaque collectivité territoriale se doit de respecter ces prescriptions, afin de participer à l'autonomie alimentaire de la Chine (Bénazéraf et Henriot, 2014). Entre 2001 et 2006, la question de l'ouverture des terres agricoles à l'urbanisation, même durable, du secteur de Dongtan n'a jamais été tranchée. Tant que Chen Liangyu soutenait le projet auprès du gouvernement central, la SIIC espérait un changement du statut du sol. Mais le limogeage de Chen Liangyu a rendu impossible cet arrangement en 2006 (Wu, 2012). Sur les 86 km² $^{2}$ initiaux, seuls $12,5 \mathrm{~km}^{2}$ pourraient être urbanisés, répondant ainsi aux exigences de préservation des sols agricoles. Pour montrer sa bonne volonté, la Société de développement de Dongtan a aménagé une zone d'agriculture biologique de $67 \mathrm{~km}^{2}$ et cherche des investisseurs pour aménager les $12,5 \mathrm{~km}^{2}$ constructibles.

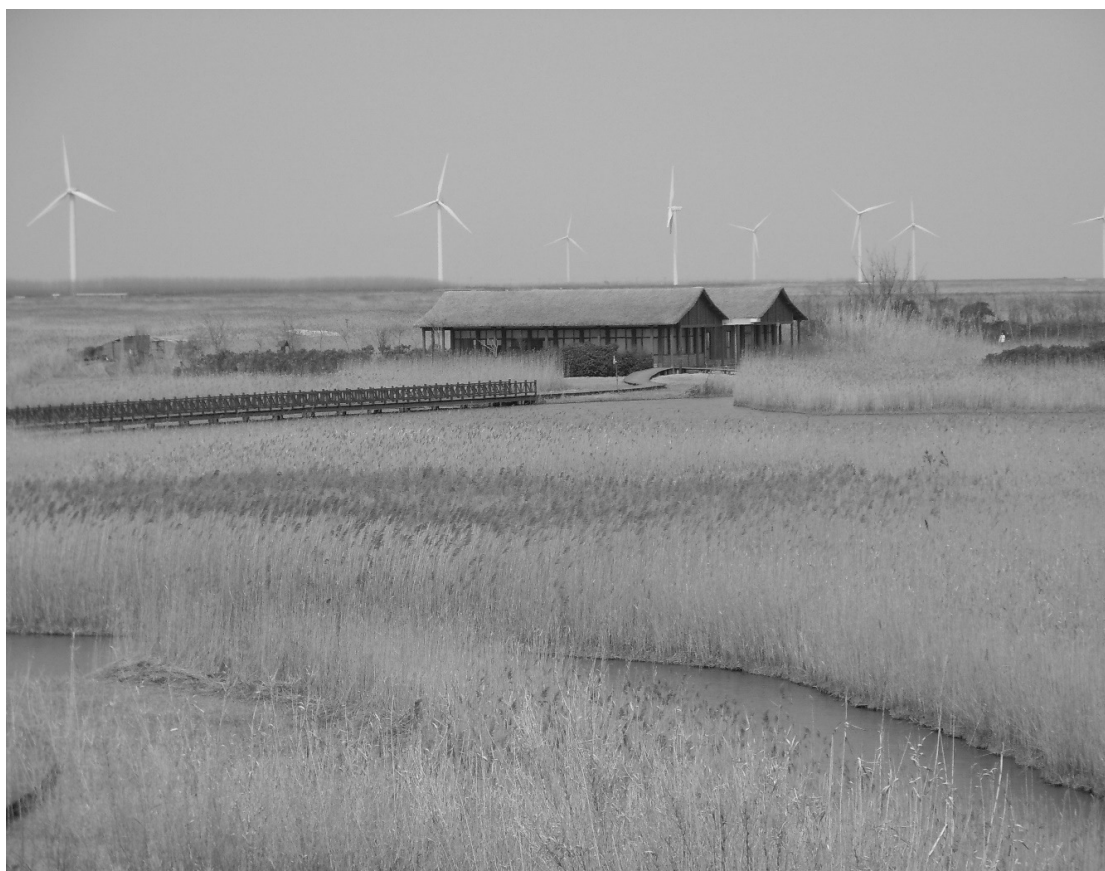

Pour autant, devant les difficultés rencontrées par le projet, les investisseurs, soucieux que leur image ne soit pas associée à l'ancien dirigeant déchu, se désengagent du projet. Un projet d'aménagement doit sa réalisation et la longévité des crédits financiers et moraux qui lui sont alloués, à la longévité du "réseau d'influence " (guanxi) porteur du projet d'aménagement. En cas de limogeage de la tête porteuse du guanxi, le projet est abandonné. Ce projet et son réseau d'influence ne sont jamais réappropriés par le nouveau dirigeant, qui souhaite avant tout favoriser les intérêts et les projets de son propre réseau. En Chine, sans continuité politique, il n'y a pas de continuité des projets d'aménagement.

Par ailleurs, le surcoût de la construction, lié au recours à des matériaux et à des technologies innovantes, n'avait pas été chiffré. Avec la crise financière de la fin 2008 et devant l'obligation de n'aménager que $12,5 \mathrm{~km}^{2}$ d'éco-ville, il est difficile, même pour la SIIC, I'une des plus grosses sociétés municipales d'investissements de Shanghai, de mobiliser des partenaires financiers. Cela se répercuterait également sur les acquéreurs de logements de l'éco-ville, lesquels ne sont pas forcément prêts à payer un tel surcoût, alors que, pour le plus grand nombre, une ville écologique est une ville dotée d'espaces verts, pas d'une ingénierie visant des objectifs en termes d'empreinte énergétique et de bilan carbone.

Enfin, le district de Chongming planifie son propre projet d'île écologique (Chongming EcoIsland Construction Outline, 2010), tandis que le bourg de Chenjia situé à proximité immédiate de Dongtan a aménagé son propre village écologique et éco-touristique à Yindong et ouvert à la promotion immobilière les secteurs attenants ${ }^{4}$.

Ainsi l'aménagement du secteur de Dongtan constitue un exemple emblématique des ajustements récents de l'action publique urbaine shanghaienne, de la diversification de ses acteurs publics, privés et internationaux, de l'association du privé au public via les sociétés de développement, de la mise en concurrence des collectivités territo-
Photo 1 : le parc de récréation de la zone humide de Dongtan, photo prise le 12/03/2011 par I'auteur.

4 - Visites de terrain le $15 / 03 / 2008$ et le 12/03/2011. 
riales municipales, de districts et de bourgs dans la maîtrise du foncier et la captation de sa rente.

\section{L'ACTION PUBLIQUE URBAINE CHINOISE : TRAJECTOIRE ET AJUSTEMENTS}

La Chine présente deux caractéristiques qui soulignent, voire isolent au sens propre du terme, son action publique urbaine. En 1949, à la création de la République populaire de Chine, les dirigeants communistes imposent l'exercice d'une réorganisation centralisatrice des territoires urbains et ruraux : rien $n^{\prime}$ existe en dehors de l'action publique communiste. Les villes chinoises sont alors mises à l'écart des politiques publiques de développement, et ce durant une quarantaine d'années. Puis, après la réintroduction progressive du marché dans la production urbaine, ces mêmes villes chinoises sont projetées dans la globalisation au tournant des années 2000 ; elles connaissent alors de profondes et rapides recompositions territoriales, orchestrées par des ajustements du rôle entre acteurs publics et acteurs privés émergents.

La trajectoire de l'action publique urbaine chinoise se déploie donc selon plusieurs phases. Chacune correspond à la mise en place de modèles de villes nouvelles variés. Les premières, vouées à la décongestion, sont élaborées dans un contexte $d^{\prime}$ industrialisation soutenue et se multiplient, jusqu'à la fin des années 1980, sous la forme de villes-satellites industrielles et $d$ 'habitat ouvrier. Plus récemment, avec l'accélération de I'urbanisation, de nouvelles dynamiques de recompositions territoriales apparaissent en périphérie des très grandes villes chinoises. Celles-ci voient la création de nouveaux quartiers urbains, dont certains correspondent à des opérations d'urbanisme de grande envergure et à des villes nouvelles censées rationaliser I'expansion périphérique et opérer un redéploiement métropolitain polycentrique vers des pôles secondaires de desserrement. Enfin, I'intégration croissante de la Chine au processus de globalisation et sa participation aux discussions internationales sur la protection de l'environnement, le développement urbain durable et la lutte contre les effets du changement climatique, engagent les villes chinoises vers de nouveaux défis. Trois modèles chinois de villes durables sont actuellement explorés : la ville écologique ou ville verte, l'éco-ville et la ville bas-carbone sont ainsi représentatives de l'inflexion de l'action publique urbaine vers une transition écologique et énergétique.

L'action publique urbaine se caractérise également par une seconde inflexion : la diversification des acteurs publics, la multiplication d'acteurs étrangers et leur mise en concurrence. À une action publique urbaine descendante et des politiques portées par l'État central et relayées par les provinces ou les municipalités se substituent des politiques de villes nouvelles et durables, portées par l'État, les collectivités territoriales - provinciales, municipales et d'arrondissement - ainsi que des acteurs gouvernementaux étrangers, dans le cadre de coopérations internationales.

Globalement, I'action publique urbaine chinoise suit les révisions des actions publiques urbaines aux Suds : tant dans sa trajectoire, avec l'évolution des orientations sectorielles et des enjeux territoriaux fixés par les politiques publiques, avec la mobilisation systématique du sol, que dans ses ajustements les plus récents, notamment la renégociation des rôles entre public et privé. Dans ce dispositif, le modèle de la ville nouvelle fait luimême trajectoire et ses inflexions témoignent de la révision de l'action publique chinoise, tout en suivant les conditions générales des actions publiques urbaines aux Suds et aux Nords, avec la prise en compte des enjeux du développement urbain durable.

L'action publique urbaine chinoise et sa trajectoire se distinguent toutefois par un contexte de forte intervention publique, un contexte autoritaire, où tout, à commencer par l'urbain, reste sous contrôle. Les réformes de la gouvernance $n$ 'ont pas abouti à une transition démocratique, mais à la réaffirmation de l'encadrement de l'État sur les territoires urbains et ses populations.

\section{BIBLIOGRAPHIE}

ANTIER G. et ABADIA G., 1987, La longue marche de Shanghai, Cahiers de I'IAURIF, $\mathrm{n}^{\circ} 81$, p. 49-58.

ANTIER G., 1989, Villes nouvelles, extérieur nuage, Cahiers de I'IAURIF, n' ${ }^{\circ}$ 87-88. p. 139-145.

BAEUMLER A., IJJASZ-VASQUEZ E., MEHNDIRATTA S., 2012, Sustainable LowCarbon City Development in China, World Bank Publications, $588 \mathrm{p}$.

http://siteresources.worldbank.org/ EXTNEWSCHINESE/Resources/

$3196537-1202098669693 / 4635541$. 1335945747603/low_carbon_city_full_en.pdf

BANQUE ASIATIQUE DE DÉVELOPPEMENT, 2010, Sustainable Urban Development in the People's Republic of China. Eco-City Development - a new and sustainable way forward?, Urban Innovations and best Practices, $8 \mathrm{p}$.

BANQUE MONDIALE, 2009, Sino-Singapore Tianjin Eco-City : A case study of an Emerging Eco-City in China, Technical Assistance Report, World Bank Publications, $142 \mathrm{p}$.

BAUDON L., 2002, Mutations de l'espace urbain à Shanghai : une mégapole entre ville globale 
et culture locale, Bulletin de I'Association des Géographes Français, vol. 79, n 4, p. 375-388.

BÉNAZÉRAF D. et HENRIOT C., 2014. La nouvelle réforme foncière chinoise : rationaliser l'urbanisation en libéralisant le foncier, China Analysis, $\mathrm{n}^{\circ} 48$, p. 46-50.

URL : http://www.centreasia.eu/publication/ china-analysis-n48-benazeraf-henriot-nouvellereforme-fonciere-chinoise

BERGÈRE M.-C., 2002, Histoire de Shanghai, Paris, Fayard, 520 p.

BILLARD G. et al., 2005, Riverside, anatomie d'une ville nouvelle chinoise, Les Annales de la Recherche Urbaine, $\mathrm{n}^{\circ}$ 98, Paris, Centre de recherche et d'urbanisme, p. 159-166.

BUREAU DES STATISTIQUES DE SHANGHAI, 2015, 2014 Nian shanghaishi guomin jingji he shehui fazhan tongji gongbao, publié le 28/02/2015, consulté le 1/05/2015.

URL:http://www.stats-sh.gov.cn/sjfb/201502/ 277392.html

CHALINE C., 1985 (réed. 1996), Les villes nouvelles dans le monde, Paris, PUF, $\mathrm{n}^{\circ} 2231,127 \mathrm{p}$.

CHAN K.-W., 2010, Fundamentals of China's urbanization and policy, The China Review, vol. 10, $\mathrm{n}^{\circ} 1$, p. 79. URL: http://faculty.washington. edu/kwchan/Chan-Fundamentals-CR2010.pdf, consulté le 1/05/2015.

Chongming Eco-Island Construction Outline (2010-2020), consulté le 29/05/2015.

URL: http://www.cmx.gov.cn/cm_website/html/ eng_cmzf/Eng_cmzf_cmzf_fzjh/List/list_0.htm

CURIEN R., 2014. La planification des villes chinoises, Perspectives chinoises, $n^{\circ} 3$, p. 27-35.

DEN HARTOG H., 2010 (dir.), Shanghai new towns : searching for community and identity in a sprawling metropolis, Rotterdam, 010 Publishers, $416 \mathrm{p}$.

DOULET J.-F., 2015, L'urbanisme chinois et l'émergence du modèle " intégrationniste ", Métropolitiques.

URL : http://www.metropolitiques.eu/L-urbanisme-chinois-et-l-emergence.html

ETTEINGER B., 1992, Yi Zhuang : un projet de ville nouvelle dans la région de Beijing, Rapport de mission du 15-28/05/1992, réalisé pour I'IAURIF, $41 \mathrm{p}$.

FRIEDMANN J., 2006, Four theses in the study of China's urbanization, International Journal of Urban and Regional Research, vol. 30, $\mathrm{n}^{\circ} 2$, p. 440-451.
FUNG K.-I., 1981a, The spatial development of Shanghai, in HOWE C., 1981, Shanghai. Revolution and development in an Asian metropolis, Cambridge, Cambridge University Press, p. 269-300.

FUNG K.-I., 1981b, Satellite town development in the Shanghai city region, Town Planning review, vol. $52, n^{\circ} 1$, p. 26-46.

FUNK K.-I., 1996, Satellite towns: development and contributions, in YEUNG Y.-M. et SUNG Y.W. (dir.), Transformation and modernization under China's Open Policy, Hong Kong, The Chinese University Press, p. 321-340.

GED F, 2000, Shanghai, Paris, Institut français d'architecture, $64 \mathrm{p}$.

[Gouvernement de la municipalité de Shanghai] Shanghai renmin zhengfu, 2011, Shi'erwu guihua yu fazhan : rang chengshi shengtai gengjia qingxin yiren [XII e programme d'action et de développement quinquennal : rendre l'environnement de la ville plus propre et plus plaisant], http:// www.shanghai.gov.cn/shanghai/node $2314 /$ node25307/node25455/node25474/node25481/ u21 ai481990.html, mis en ligne le 17/02/2011 et consulté le 1/05/2015.

HENRIOT C., 2013a, Aménager les périphéries urbaines chinoises : ville nouvelle et partenariat public-privé à Shanghai, URBIA. Les cahiers $d u$ développement urbain durable, hors-série $\mathrm{n}^{\circ} 1$ « Urbanisme et aménagement du territoire, un aperçu de la jeune recherche francophone ", p. 207-222.

HENRIOT C., 2013b, Villes nouvelles et redéploiement métropolitain à Shanghai. Les nouvelles périphéries urbaines chinoises, thèse de doctorat en géographie, université Paris 1 PanthéonSorbonne, $440 \mathrm{p}$.

HENRIOT C., 2014, Les logements sociaux du "Nouveau Triomphe " à Shanghai : facteurs de fragmentation sociale et spatiale, in BELMESSOUS F., BONNEVAL L., COUDROY DE LILLE L., ORTAR N., Logement et politique(s). Un couple encore d'actualité ?, Paris, L'Harmattan, p. 85-102.

HENRY M., 1984, Pékin, Shanghai et la région île-de-France, un début d'échange d'informations, Cahiers de I'IAURIF, n073, p. 65-70.

HOA L., 1981, Reconstruire la Chine, trente ans d'urbanisme, 1949-1979, Paris, Le Moniteur, p. 63-65.

HOUSSAY-HOLZSCHUCH M., 2010, Crossing boundaries : Vivre ensemble dans I'Afrique du Sud post-apartheid, HDR, Université Paris 1 PanthéonSorbonne, tome 3, $270 \mathrm{p}$. 
HUANG B. et al., 2008, Construction of an ecoisland : a case study of Chongming Island, China, Ocean and Coastal Management, ${ }^{\circ}{ }^{51,}$ p. 576.

KIRKBY R.J.R., 1987, A review of satellite town policies in the People's Republic of China. The experience of Shanghai, in PHILIPS D. et YEH A. (dir.), 1987, New towns in East and South East Asia, Hong Kong, Oxford University Press, p. 205-230.

LEWIS A., 1954, Economic Development with Unlimited Supply of Labour, The Manchester School of Economic and Social Studies, vol. 47, n³, p. 139-191.

LIN G. et D. WEI, 2002, China's restless urban landscapes: new challenges for theoretical reconstruction, Environment and Planning A, vol. 34, $\mathrm{n}^{\circ}$ 9, p. 1535-1536.

LORRAIN D., 2010, "Portrait d'entreprise " Blue red chips: les compagnies d'eau en Chine, Flux, $n^{\circ} 82$, p. $64-80$.

LORRAIN D., 2011, Shanghai ou une modernisation publique, in LORRAIN D. (dir.), Métropoles $X X L$ en pays émergents, Paris, Les Presses de Science Po, p. 53-138.

MA L.J.C., 2002, Urban transformation in China, 1949-2000 : a review and research agenda, Environment and planning $A$, vol. 34, $\mathrm{n}^{\circ} 9$, p. 1545-1569.

MERLIN P., 1972, L'urbanisme en région de Londres et les villes nouvelles, in MERLIN P., Les villes nouvelles. Urbanisme régional et aménagement, Paris, Presses universitaires de France, p. 11-82

MERLIN P., 1996, Ville nouvelle, in MERLIN P. et CHOAY F., Dictionnaire de l'urbanisme et de l'aménagement, Paris, PUF, p. 847-850.

MONIN É., 2012. Figure 1. Aires métropolitaines et schéma d'aménagement écologique de la municipalité, in "Trames du delta du Yangzi : recompositions métropolitaines et aménagement des périphéries agricoles de Shanghai ", Projets de paysage, $\mathrm{n}^{\circ} 8$.

URL : http://www.projetsdepaysage.fr/trames du delta du yangzi recompositions metropolitaines_et_amenagement_des_peripheries_agricoles de $\bar{d}$ shanghai chine

NING Y. et YAN Z., 1995, The changing industrial and spatial structure in Shanghai, Urban Geography, vol. $16, \mathrm{n}^{\circ} 7$, p. 577-594.

PANNELL C., 2002, China's continuing urban transition, Environment and Planning A, vol. 34, n०9, p. 1571-1589.

PETIT O., 2002, L'influence des villes nouvelles françaises en Asie, dans leur rapport avec les idées, les entreprises et les hommes de l'art français, Programme interministériel d'histoire et d'évaluation des villes nouvelles françaises, La Défense, ISTED "Villes en développement ", $115 \mathrm{p}$.

SANJUAN T., 2000, La Chine. Territoire et société, Paris, Hachette, 189 p.

SHANGHAI TONGJI CHENGSHI GUIHUA SHEJI YANJIU YUAN [Institut d'urbanisme de Tongji à Shanghai], 2011, Shi'erwu shanghai jiaoqu xincheng guihua [Planification des villes nouvelles du XII ${ }^{e}$ programme d'action quinquennal de Shanghai], p.4.

SHEN J. et WU F., 2012, Restless urban landscapes in China : a case study of three projects in Shanghai, Journal of Urban Affairs, vol. 34, $\mathrm{n}^{\circ} 3$, p. 236.

WANG Z. et LI Z., 2006, "Yi cheng jiu zhen», duijiaoqu xinchengzhen de qishi, [Éclaircissements sur les bourgs nouveaux de banlieue du programme "une ville, neuf bourgs»], Jianzhu xuebao [Architectural Journal], $n^{0} 7$, p. 8-11.

WANG Y., 2011, Xin Jiangwan, "éco-quartier' à Shanghai", Espace chinois - urbains et culture, Eurorient, $\mathrm{n}^{\circ}$ 33-34, L'harmattan, p. 133-152.

WU F., 2012, China's ecocities, Geoforum, vol. $43, n^{\circ} 2$, p. 169-171.

ZHOU N., HE G. and WILLIAMS C., 2012, China's Development of Low-Carbon Eco-Cities and Associated Indicator Systems, Rapport de recherche rédigé pour China Energy Group, Energy Analysis \& Environmental Impacts Department, Environmental Energy Technologies Division, Ernest Orlando Lawrence, Berkeley National Laboratory.

URL: http://china.Ibl.gov/sites/all/files/china_ecocities_indicator_systems.pdf

YU S. et LUO Z. 2009, Shanghai jiaoqu xincheng de guihua yu sikao [Planification et réflexions sur les villes nouvelles de la banlieue de Shanghai], Chengshi guihua xuekan [Cahiers d'urbanisme], vol. 3, nº181, p. 13-19.

ZANG M., 2012, Dongtan shengtai cheng ruhe bimian «kongcheng» ganga [La ville écologique de Dongtan ou comment éviter le phénomène de ville fantôme], Dongfang zaobao [Journal de l'Est], du 20/02/2012, consulté le 1/05/2015.

URL : http://www.dfdaily.com/html/21/2012/2/ 20/745991.shtml 
ZHANG S. et al., 2015, PPP application in infrastructure development in China: Institutional analysis and implications, International Journal of Project Management, vol. 33, p. 497-509.

ZHANG Y. et al., 2014, From State to Market: Private Participation in China's Urban Infrastructure Sectors, 1992-2008, Word Development, vol. 64 , p. $473-486$.
ZHUO J., 2004, Mobilité urbaine en Chine : enjeux et problématiques. Le cas de Shanghai et $d^{\prime}$ 'autres grandes métropoles, conférence MUTA, Mont Tremblant, N.R.

ZHUO J., 2015, TOD, un paradigme urbanistique à l'épreuve de la ville chinoise : le cas de Shanghai, Rencontres internationales de I'APERAU, Rennes, $1-5 / 06 / 2015$ 